\title{
(Digitale) Medienkompetenz in der Stadtbibliothek Kreuztal - Ein Blick in das Projekt „Wissen, Können,
} Machen"

Zusammenfassung: Digitale Medienkompetenz ist weiterhin ein wichtiger Faktor in der Arbeit einer Öffentlichen Bibliothek. Die Stadtbibliothek Kreuztal hat ein Angebot entwickelt, das alle Altersgruppen erreichen soll und dessen Fokus es ist, digitale Medien nicht nur zu konsumieren, sondern sie zu verstehen, selbst zu gestalten und einen Bezug zu ihnen herzustellen. Die Voraussetzungen, Motivationen, Angebote und Umsetzungsstrategien werden im Folgenden beschrieben.

Schlüsselwörter: Digitalisierung; Medienkompetenz; digitale Medienkompetenz

(Digital) Media literacy in the public library in Kreuztal - A closer look at the project "knowing, being capable, doing"

Abstract: Digital media literacy continues to be an important part of a public libraries work. The public library in Kreuztal developed a project, which is supposed to reach all age groups and which's focus is not to just consume digital media, but to understand, produce and to develop a closer relationship with them. The conditions, motivations, activities and implementation will be covered in the following article.

Keywords: Digitalization; media literacy; digital media literacy

\section{Gesellschaftliche Voraussetzungen}

Dass der digitale Wandel in vollem Gange ist, wird von niemandem angezweifelt. Auch dass Deutschland im Ländervergleich oftmals hinterher hinkt und bereits wie im Vorjahr im Bereich Glasfaserkabelausbau auf Platz $72^{1}$ der Rangliste des Weltwirtschaftsforums (WEF) zur Wettbewerbsfähigkeit landet, soll an dieser Stelle kein großes Thema sein, muss allerdings aufgrund

1 vgl. World Economic Forum (2019a). 
Preprints der Zeitschrift BIBLIOTHEK - Forschung und Praxis, 2020, AR 3347 Schenkschuck und Flecke

Dies ist ein Preprint (cc) EY-NG-ND. Die endgültige Publikationsfassung erscheint beim Verlag De Gruyter unter https://www.degruyter.com/view/j/bfup

der Relevanz angesprochen werden. Stattdessen richten wir unseren Blick an dieser Stelle einmal auf 6.05 Digital skills among active population. Die Frage, die das WEF an dieser Stelle an die Teilnehmenden gerichtet hat, lautete:

„In your country, to what extent does the active population possess sufficient digital skills (e.g. computer skills, basic coding, digital reading)? [1 = not all; 7 = to a great extent $]^{\text {“2 }}$

2018 erreichte Deutschland an dieser Stelle Platz 16, mit einem durchschnittlichen Ergebnis von 5.2. ${ }^{3}$ Ein Jahr später, in dem veröffentlichten Ranking für 2019 war Deutschland auf Platz 21 gefallen, und fand sich lediglich bei 5.1 wieder. Der Score (auf einer Skala von $0-100$ ) fiel von $70.2^{4}$ auf $67.8 .{ }^{5}$ Das Ergebnis selbst ist nur geringfügig schlechter geworden, steht allerdings im Kontrast zu einer Pressemitteilung des Bundesministeriums für Wirtschaft und Energie, die verlauten lässt, dass „vor allem beim Zugang zum Internet, dem Nutzungsverhalten und bei der Digitalen Kompetenz [...] bedeutsame Fortschritte" ${ }^{\prime 6} \mathrm{zu}$ beobachten sind. Analysiert wurde der Digitalisierungsgrad der deutschen Bevölkerung durch die Initiative D21 e.V. mit Unterstützung von Kantar TNS anhand der Kriterien Zugang, Kompetenz, Offenheit sowie Nutzungsvielfalt. Die Generation 50+ ist mit 8\% Punkten besonders stark gewachsen. ${ }^{7}$ Beide Statistiken fragen also verschiedene Kriterien ab, die zu den schlussendlichen unterschiedlichen Ergebnissen führen.

Fakt ist, dass die Zahl der Nutzenden digitaler Dienste wächst; Generation Z ${ }^{8}$ bis Baby Boomer ${ }^{9}$ sind digital unterwegs und werden immer wieder mit technologischem Fortschritt und neuen Medien konfrontiert. Die Schnelllebigkeit unserer Gesellschaft und des digitalen Wandels führt allerdings auch dazu, dass die Möglichkeiten, die sich durch diese Medien bieten, sowie das Erlernen des richtigen Umgangs oftmals nicht voll ausgeschöpft werden.

An dieser Stelle, können Bibliotheken die Kluft überbrücken, die durch den rasanten technologischen Wandel entstanden ist. Medien wie Games, VR Brillen, 3 Drucker, Tonies, Tablets, etc. finden sich bereits seit längerer Zeit in (Öffentlichen) Bibliotheken wieder und erfreuen sich großer Beliebtheit, nicht nur bei jüngeren Menschen. Dass (Öffentliche) Bibliotheken daher nicht erst seit kurzem digitale Medien in ihr Angebot einbauen, sowohl um Medienkompetenz zu vermitteln, Spaß am Spiel

\footnotetext{
${ }^{2}$ World Economic Forum (2019b).

${ }^{3}$ vgl. World Economic Forum (2018).

${ }^{4} \mathrm{vgl}$. ebd.

${ }^{5}$ vgl. World Economic Forum (2019b).

${ }^{6}$ Bundesministerium für Wirtschaft und Energie (2020).

${ }^{7}$ vgl. Bundesministerium für Wirtschaft und Energie (2020).

${ }^{8}$ Menschen, die zwischen den Jahren 2000 und 2019 geboren wurden.

${ }^{9}$ Menschen, die zwischen den Jahren 1946 und 1964 geboren wurden.
} 
Preprints der Zeitschrift BIBLIOTHEK - Forschung und Praxis, 2020, AR 3347 Schenkschuck und Flecke

Dies ist ein Preprint (cc) EY-NG-ND. Die endgültige Publikationsfassung erscheint beim Verlag De Gruyter unter https://www.degruyter.com/view/j/bfup

zu wecken, oder auch beide Punkte miteinander zu verbinden, ist kein Geheimnis und auch keine Neuheit mehr. Vielen gelingt dies auch bereits mit großem Erfolg.

\section{Motivation für den Ausbau des Angebots}

In der Stadtbibliothek Kreuztal werden Medienkompetenz und digitale Angebote bereits seit mehreren Jahren mit großer Resonanz angeboten. Vom Bilderbuchkino, Führungen, über Gamingnachmittage und -turniere, hat sich die kleine Bibliothek - Kreuztal verfügt über 30000 Einwohner, die Bibliothek über 40000 Medien, sowie 8 Mitarbeiterinnen auf 5,4 Personalstellen bereits sehr breit aufgestellt.

Durch einen personellen Wechsel Ende 2018 und Anfang 2019 wurden die bereits bestehenden Angebote unter der Aufsicht der neuen Bibliotheksleitung Linda Donalies weiter ausgebaut, neu verteilt und überdacht. Das Ziel der neuen Veranstaltungskonzepte war dabei ganz klar:

- mehr Teilnehmende,

- aktuellere Themen und

- nachhaltigere Resultate.

Die Stadtbibliothek Kreuztal ist Anlaufstelle für eine große Anzahl von Jugendlichen, die die Bibliothek als Aufenthaltsraum nutzen, nachmittags FIFA spielen und das WLAN nutzen. Viele von innen haben wenig Interesse an klassischen Angeboten der Leseförderungen und beschäftigen sich lieber mit digitalen Medien, können dann allerdings, wenn sie vor einem Problem stehen, nicht mit ihnen umgehen oder gehen in ihren Handlungen nicht über das Konsumieren hinaus. Oftmals wahrscheinlich, weil es ihnen von Haus oder auch aus der Schule hinaus an Möglichkeiten fehlt, sich differenziert mit den Medien auseinander zu setzen, da es Eltern, Großeltern und Lehrbeauftragten schlicht und einfach an Zeit mangelt, sich mit trivialen Medien zu beschäftigen zu setzen. Die Schnelllebigkeit und der Leistungsdruck der Gesellschaft spielen auch hier eine große Rolle. Es mussten also Veranstaltungen her die a) die Jugendlichen dazu befähigen kompetenter mit Medien umzugehen b) Kindern bereits im jungen Alter den Spaß am aktiv Mitwirken und Verstehen von Medien vermitteln um eine Grundlage zu schaffen, sowie c) Eltern und auch der älteren Generation die Möglichkeit geben, sich mit Medien vertraut zu machen, die für sie schlecht zugänglich sind.

Als neue Leiterin der Kinder- und Jugendbibliothek entwickelte Sabine Flecke ab Ende 2018 eine Reihe von verschiedenen Angeboten, die im Folgenden vorgestellt werden und sich im speziellen an 
Preprints der Zeitschrift BIBLIOTHEK - Forschung und Praxis, 2020, AR 3347 Schenkschuck und Flecke

Dies ist ein Preprint $(\boldsymbol{c c})$ EY-NG-ND . Die endgültige Publikationsfassung erscheint beim Verlag De Gruyter unter https://www.degruyter.com/view/j/bfup

Kinder richten, um die unter b) genannten Ziele anzugehen. Bereits existierende Angebote für Jugendliche und Eltern wurden von Annamarie Escher-Schenkschuck weiter ausgebaut.

Das Wissen, welches für die Vermittlung der relevanten Fähigkeiten und Kompetenzen nötig ist, eigneten sich die Kolleginnen selbst an, getrieben von unterschiedlichen Motivationen. Frau Flecke wollte insbesondere die Neugier und den natürlichen Wissensdurst der Kinder wecken und sie animieren, sich aktiv mit Medien und Mintthemen zu beschäftigen. Sie sollten dazu angeleitet werden, selbst zu produzieren anstatt lediglich zu konsumieren. Frau Escher-Schenkschuck wollte Menschen jeden Alters die Möglichkeit geben, am digitalen Leben teilzunehmen, und dieses zu verstehen, sodass sie die Medien, die sie nutzen nicht zur konsumieren, sondern auch analysieren können.

Durch die Ziele, die Linda Donalies sich für die Stadtbibliothek gesetzt hatte, war es möglich, die Angebote der Bibliothek zu einem stimmigen Konzept zusammen zu setzen, das schlussendlich in dem Projekt: Wissen, Können, Machen mündete.

\section{$3 \quad$ Aktionen im Projekt: Wissen, Können, Machen}

Das Projekt bietet den Teilnehmenden die Möglichkeit, neue Medien nicht nur zu konsumieren, sondern aktiv an ihnen mitzuwirken und sie zu verstehen. Die einzelnen Veranstaltungen sind darauf ausgelegt, die Teilnehmenden zu aktivieren. Durch das Wissen, welches ihnen von den Kolleginnen vermittelt wird, ist es ihnen möglich Medien selbst zu gestalten, sowie einen Teil zur Medienkompetenz der Gesellschaft beizutragen.

Dies wird durch eine Reihe verschiedener Angebote mit gleicher Zielsetzung gewährleistet, die sich stimmig in das Gesamtkonzept der Bibliothek einfügen. Sie werden von unterschiedlichen Kolleginnen der Bibliothek durchgeführt.

\section{Kreatives Arbeiten mit Medien: Erstellen von}

- TipToi Büchern

- Bilderbuchfilmen

- Hörbüchern

- eBooks

Schulen und Kindergärten haben die Möglichkeit unter Anleitung von Frau Flecke Medien selbst zu gestalten. Die Texte und Bilder werden von den Kindern selbst erstellt. Dabei kann es sich sowohl um selbstgemalte Bilder als auch um Fotos handeln. Unter Anleitung der Kollegin werden die Texte von den Kindern selbst gesprochen und Geräusche erzeugt und aufgenommen. Die weitere technische 
Preprints der Zeitschrift BIBLIOTHEK - Forschung und Praxis, 2020, AR 3347 Schenkschuck und Flecke

Dies ist ein Preprint $(\boldsymbol{c c})$ EY-NG-ND . Die endgültige Publikationsfassung erscheint beim Verlag De Gruyter unter https://www.degruyter.com/view/j/bfup

Bearbeitung wird von Frau Flecke übernommen, kann bei älteren Kindern aber auch von diesen selbst erlernt werden. Die Medien werden bei einer Premiere in der Stadtbibliothek vorgeführt und im Anschluss den Schulen und Kindergärten übergeben. Ziel der Aktion ist es, die Kinder wegzuführen vom bloßen Konsumieren hin zum Produzieren, ihnen Einblicke zu geben in die Herstellung von Medien und ihre Freude an verschiedenen Formaten von Literatur zu wecken.

MINT Förderung: Im Rahmen der MINT Förderung werden naturwissenschaftliche und technische Themen in einzelnen Veranstaltungen und auf Anfrage auch für Schulen und Kindergärten behandelt und praktisch umgesetzt. Unter Einbezug des Sachbuchbestandes werden unter Anleitung von Frau Flecke beispielsweise Putzroboter, Krabbelroboter, eine Wetterstation, LED Lesezeichen, LED Armbänder und Solarboote selbst gebaut, hergestellt und Roboter programmiert. Das Ziel der Veranstaltungen ist, das naturwissenschaftliche und technische Verständnis der Kinder zu fördern, den Umgang mit digitalen Programmen und Medien zu erlernen, ein Verständnis für das Programmieren zu vermitteln und sich somit aktiv auf die fortschreitende Digitalisierung im privaten und wirtschaftlichen Bereich vorzubereiten.

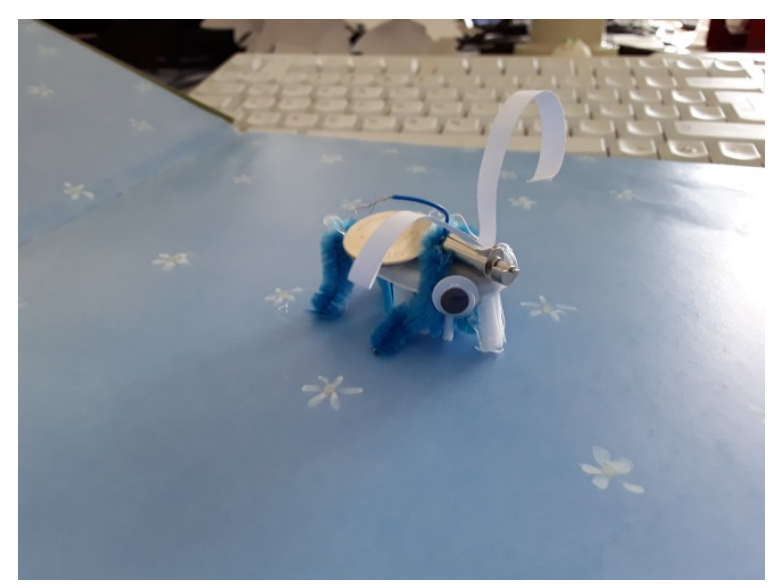

Abb. 1: Im Rahmen der MINT Förderung bauen die Kinder selbst Putzroboter Sonntagsaktionen: Jeden ersten Sonntag im Monat werden den Besuchenden der Aktion unterschiedliche Themengebiete und neue Formate näher gebracht, die von ihnen, nach einer Einführung, selbst umgesetzt werden. Die Veranstaltungen werden von den Mitarbeiterinnen selbst, aber auch von externen lokalen Unternehmen, regionalen Vereinen, sowie Privatpersonen unterstützend durchgeführt.

Dazu zählen:

- Das Ausprobieren von VR-Technologie, sowie 3D Druckern,

- Salz aus Frühlingskräutern herstellen,

- Lego-Roboter bauen und Bee-Bots programmieren, 
Preprints der Zeitschrift BIBLIOTHEK - Forschung und Praxis, 2020, AR 3347 Schenkschuck und

\section{Flecke}

Dies ist ein Preprint $(\mathrm{cc})$ EY-NG-ND. Die endgültige Publikationsfassung erscheint beim Verlag De Gruyter unter https://www.degruyter.com/view/i/bfup

- gemeinsam tanzen unter Anleitung des lokalen Tanzklubs Casino,

- gemeinsames Spielen und Vernetzen untereinander beim Pen\&Paper Rollenspiel Nachmittag mit Dungeons\&Dragons

- Puppentheater und Bilderausstellung der Jugendkunstschule und Musikschule,

- Näherbringen von naturwissenschaftlichen Themen durch Mitmachexperimente,

- einen Gedächtnisparcours absolvieren,

- Origamifalten mit den Teilnehmer*innen von Origami Deutschland e.V.,

- Vögel kennen, Vögel schützen: digitales Vogelquiz + Raubvogelsilhouetten für Fenster basteln.

Die Sonntagsaktion strebt an, Neues in einem zwanglosen Rahmen ohne Zeit- oder Leistungsdruck auszuprobieren und zukünftige Angebote, wie VR-Technologie, 3D Drucker, sowie

Dungeons\&Dragons einführend vorzustellen.

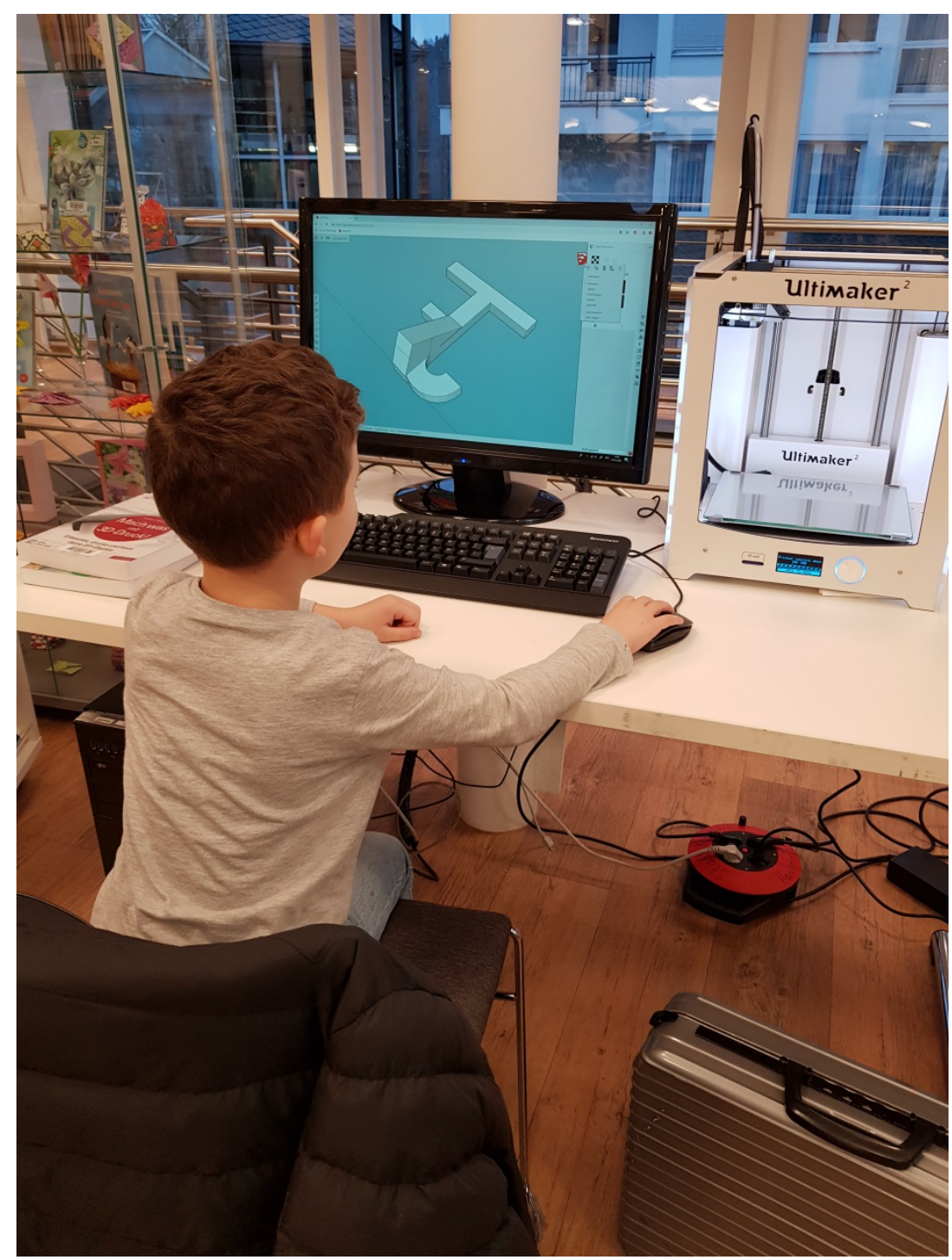


Preprints der Zeitschrift BIBLIOTHEK - Forschung und Praxis, 2020, AR 3347 Schenkschuck und Flecke

Dies ist ein Preprint (cc) EY-NG-ND. Die endgültige Publikationsfassung erscheint beim Verlag De Gruyter unter https://www.degruyter.com/view/j/bfup

Abb. 2: Bei einer Sonntagsaktion lebt dieser Bibliotheksbenutzer seine kreative Seite an einem 3D Drucker aus

Spieletester: In Zusammenarbeit mit der Fachstelle für Jugendmedienkultur treffen sich Kinder und Jugendliche im Alter von 12 bis 18 Jahren zweimal im Monat um verschiedene Spiele für den Computer, die Nintendo Switch oder die Playstation 4 zu testen. Im Anschluss an eine 2- 4stündige Testphase werden für diese Spiele pädagogische Bewertungen von den Jugendlichen verfasst. Ein besonderer Fokus liegt dabei auf der pädagogischen Altersbewertung, den problematischen Effekten im Bereich der Gewalt- und Geschlechterdarstellung, sowie der finanziellen Kosten und der Suchtgefahr. Diese Bewertung, die von den Jugendlichen unter pädagogischer Anleitung durch Frau Escher-Schenkschuck erstellt wird, wird auf der Seite des Spieleratgeber NRW veröffentlicht und dient Lehrkräften und Eltern als Ansatzpunkt für die Auswahl und das Verstehen der Spiele, mit denen die Kinder und Jugendlichen sich beschäftigen und die ihr Interesse wecken. Die Jugendlichen gewinnen dadurch ein Verständnis dafür, welchen Einfluss Computerspiele auf sie haben, lernen lange Texte zu verfassen, schulen ihre Medienkompetenz und tragen einen Teil zur Medienkompetenz und zum Medienverständnis andere Kinder und Jugendlichen bei.

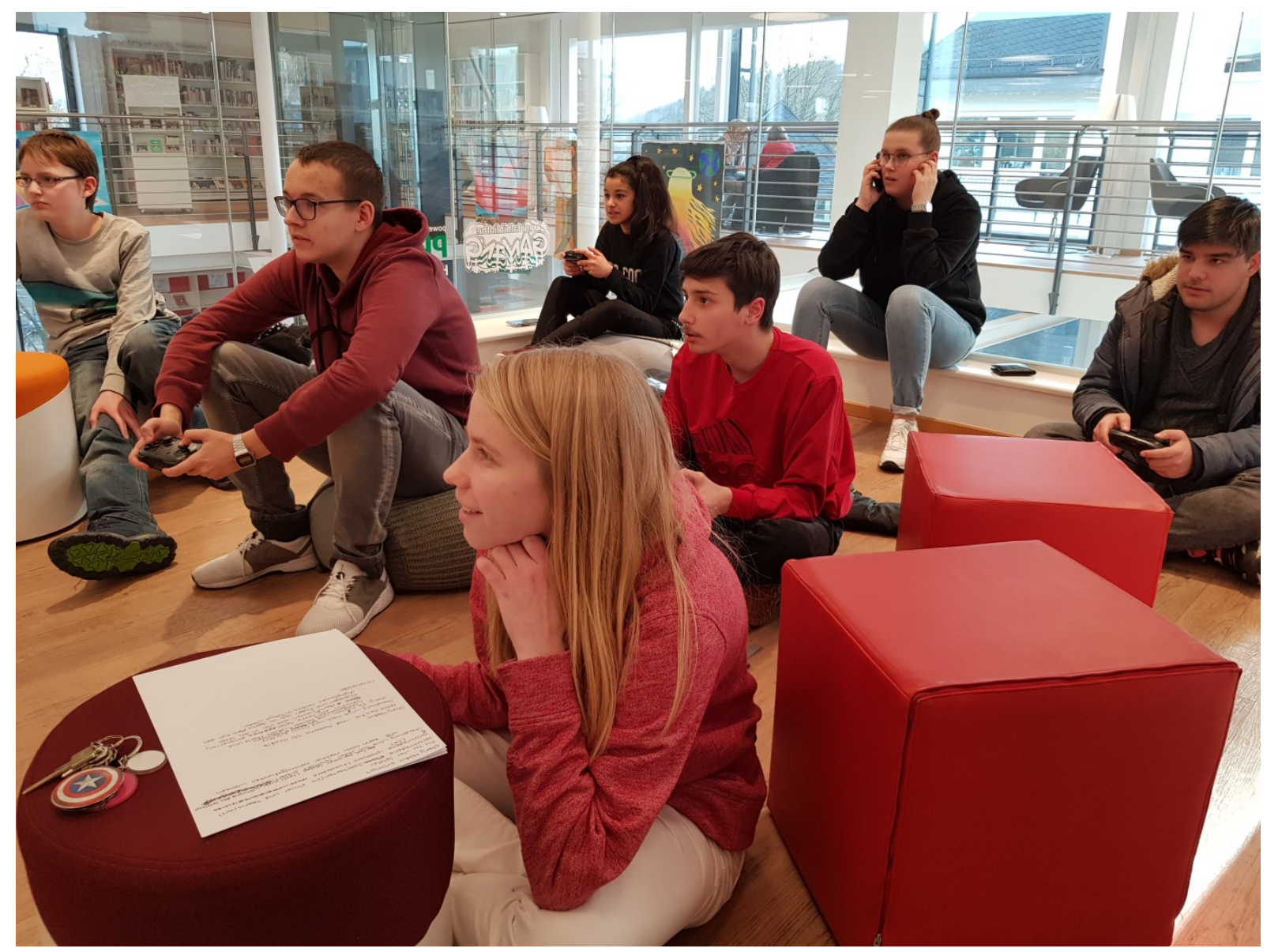


Preprints der Zeitschrift BIBLIOTHEK - Forschung und Praxis, 2020, AR 3347 Schenkschuck und Flecke

Dies ist ein Preprint (cc) EY-NG-ND. Die endgültige Publikationsfassung erscheint beim Verlag De Gruyter unter https://www.degruyter.com/view/j/bfup

Abb. 3: Die Spieletester treffen sich alle zwei Wochen in der Bibliothek um Spiele zu testen und pädagogische Bewertungen für diese zu schreiben

Computerspiele für Eltern: In einer 1,5-stündigen Abendveranstaltung wird Eltern das Thema Gaming durch Frau Escher-Schenkschuck näher gebracht. Sie erfahren, was ihre Kinder an dem Thema Gaming interessiert, welche Vor- und Nachteile entstehen, was für Spiele derzeit beliebt sind und aus welchen Gründen ihre Kinder sich dafür begeistern. Als praktische Übung wird im Anschluss selbst ein beliebtes Spiel gespielt (zuletzt Fortnite), damit die Eltern ein handfestes Gefühl für das bekommen, was sie in der Veranstaltung gehört haben. Die Eltern lernen mit Konsolen und Computerspielen umzugehen, und ihre Kinder besser zu verstehen. Ihnen werden weitere hilfreiche Informationen und Portale vermittelt, bei denen sie sich über die Gefahr, die von bestimmten Spielen ausgeht, informieren können.

Techniksprechstunde: Besonders für ältere Nutzende wird eine Techniksprechstunde angeboten, bei der verschiedene technische Aspekte und Gerätschaften erklärt, erläutert und mit ihnen zusammen eingerichtet werden. Unter anderem handelt es sich dabei um WhatsApp und seine Alternativen, Gaming, Facebook, die Onleihe, sowie verschiedene AppStores. Auf Wünsche der Teilnehmenden wird aktiv eingegangen, sodass sie die Inhalte der Veranstaltung selbst mitgestalten. Ziel der Veranstaltung ist es, den Nutzenden die Möglichkeit zu geben, den Anschluss bei der Digitalisierung nicht zu verlieren, aktiv am (digitalen) Leben teilzuhaben und ihnen einen Überblick über die vielfältigen Möglichkeiten zu geben, die sich ihnen eröffnen.

Gamingturnier: Im Gamingraum der Stadtbibliothek finden monatlich Gamingturniere zu unterschiedlichen Spielen statt. Dabei wird darauf geachtet, dass nicht nur das von den Kindern beliebte Fifa gespielt wird, sondern ihnen auch andere Spiele vorgestellt werden, die ihr Interesse vielleicht noch nicht geweckt haben. Durch das gemeinsame Spielen schulen sie ihre Teamfähigkeit, ihren Wettkampfgeist, aber auch den Umgang mit potentiellen Enttäuschungen. Durch die unterschiedlichen Spiele und Konsolen, an denen die Kinder und Jugendlichen spielen, lernen sie den Umgang mit ihnen - vielleicht unbekannten - Medien und steigern ihre Medienkompetenz im Gamingbereich.

\section{$4 \quad$ Umsetzung}

Der bereits erwähnte Personalwechsel Ende 2018 und Anfang 2019 führte zu einer Fülle an neuen Ideen und Konzepten, die nach einer Einarbeitungsphase von Frau Flecke und Frau Donalies ab Februar 2019 Gestalt annahmen. Der Aktionssonntag wurde als erste Veranstaltungsreihe zu den bestehenden Angeboten dazu genommen und erfreut sich aufgrund der thematischen Vielfalt großer 
Preprints der Zeitschrift BIBLIOTHEK - Forschung und Praxis, 2020, AR 3347 Schenkschuck und Flecke

Dies ist ein Preprint $(\boldsymbol{c c})$ EY-NG-ND . Die endgültige Publikationsfassung erscheint beim Verlag De Gruyter unter https://www.degruyter.com/view/j/bfup

Beliebtheit. Positiv zu beobachten war von Anfang an, dass sich sowohl Kinder als auch Erwachsene von den Aktionen angesprochen fühlten, sei es das Ausprobieren einer VR-Brille oder das Nähen von Lesezeichen. Im Laufe des Jahres 2019 wurden weitere Aktionen hinzugefügt, die alle unter den Zielen des Projektes standen.

Nach persönlichen Besuchen in den Schulen und Kindergärten im Umkreis zum Präsentieren der neuen Angebote MINT Förderung und Kreatives Arbeiten mit Medien bestand bald reges Interesse an den Aktionen des Projektes. Privatpersonen traten auf die Bibliothek zu, um am Aktionssonntag etwas vorzustellen, und die Bibliothek hat sich an lokale Unternehmen gewandt, um Kooperationspartner für die Aktionen zu werben. Die Resonanz war überwältigend. Die Zahl der Veranstaltungen erhöhten sich im Vergleich zu 2018 von 123 auf 166. Besucht wurden diese von 2982 Nutzenden. Im Jahr davor waren es 1585. Das Feedback der Teilnehmenden ist durchweg positiv und die Aktionen sind ein fester Bestandteil des Portfolios der Stadtbibliothek.

Die Veranstaltungen wurden neben der Website, natürlich auch in lokalen Zeitungen und mit Plakaten in Supermärkten und Buchhandlungen beworben. Ein stärkerer Fokus wurde auf Social Media - Facebook und Instagram - gerückt. Dort wurden die Aktionen mit Filmen, die zeigten, was man sich unter der Thematik vorstellen konnte, beworben. Aber natürlich fanden sich auch gängige Veranstaltungshinweise in Text und/oder Bildform wieder.

\section{Pläne und Ausblick}

Nachdem sich auch weitere Veranstaltungen außerhalb des Projektes in der Stadtbibliothek etabliert hatten - sechs verschiedene Escape Rooms und Lesekompetenz für Schulkinder - wurde die Bibliotheksstrategie im neuen Jahr direkt weiter ausgebaut und neue Ziele wurden gesetzt.

Neue Veranstaltungen sollten her. Das Team war so eingespielt und bewältigte die bestehenden Angebote, dass noch mehr Aktionen ohne Probleme hinzugefügt werden konnten.

Eine Techniksprechstunden, die bereits in der Vergangenheit angeboten worden war, sollte wieder ins Leben gerufen werden, das Gaming Turnier, welches bisher nur ein bis zweimal im Jahr veranstaltet wurde, sollte mit wechselnden Spielen monatlich angeboten werden.

2020 sollte im Zeichen der Technik und Digitalisierung stehen: Zwei VR-Brillen, ein 3D Drucker, Zeichentablets, sowie, neue Roboter zum Verleihen werden angeschafft. Diese sollen allerdings nicht nur unter Aufsicht benutzt werden können. Das Konzept sieht vor, dass die Nutzenden das Wissen von Mitarbeiterinnen erwerben und dieses dann selbstständig umsetzen und anwenden. 
Preprints der Zeitschrift BIBLIOTHEK - Forschung und Praxis, 2020, AR 3347 Schenkschuck und Flecke

Dies ist ein Preprint (cc) EY-NG-ND. Die endgültige Publikationsfassung erscheint beim Verlag De Gruyter unter https://www.degruyter.com/view/j/bfup

Bauliche Maßnahmen wurden bereits getroffen, die Fläche für einen Maker Space ist frei, die Gelder sind beantragt. Sobald diese eingetroffen sind und wieder Veranstaltungen angeboten werden können, ist die Bibliothek also für die zweite Phase der Digitalisierung bereit.

\section{$6 \quad$ Fazit}

Digitale Medien sind längst ein gängiges Thema für Nutzende von Bibliotheken. Trotzdem fehlen vielen die Fähigkeiten, mit ihnen umzugehen und mehr zu tun, als sie einfach nur zu konsumieren. Daher ist es nur natürlich, dass Öffentliche Bibliotheken ihrem Bildungsauftrag nachkommen und allen Willigen die Möglichkeit bieten, sich die Fertigkeiten und das Verständnis dafür anzueignen. Dies betrifft alle Altersgruppen, von Kindern, die ihre erste Erfahrung beim Programmieren sammeln, bis hin zur älteren Generation, die den Anschluss nicht verpassen möchte. Viele von den Veranstaltungen, die Teil des Projekts: Wissen, Können, Machen sind, lassen sich auch in anderen Bibliotheken nach einer Einarbeitung in die Technik und die Materie umsetzen.

Die Stadtbibliothek Kreuztal strebt mit ihrem Angebot an, alle Altersgruppen zu erreichen und ihnen den Zugang zur Digitalen Welt zu erleichtern, zu ebnen und sie zu begleiten. Neue Medien sollen laufend in den Alltag der Nutzenden und das Angebot der Bibliothek integriert werden, um ihnen nachhaltiges Wissen für die Zukunft zu vermitteln.

Sie trägt damit ihren Teil zu den dringend benötigten Digitalen Fähigkeiten bei, die in Zukunft keinesfalls weniger benötigt werden, sondern auf die wir uns eher immer öfter werden berufen müssen.

\section{Literaturverzeichnis}

World Economic Forum (2018): 6.05 Digital skills among active population. Verfügbar unter https://reports.weforum.org/global-competitiveness-report-2018/competitivenessrankings/?doing_wp_cron=1590397950.1625969409942626953125\#series=EOSQ508.

World Economic Forum (2019a): 3.04 Fibre internet subscriptions. Verfügbar unter https://reports.weforum.org/global-competitiveness-report-2019/competitivenessrankings/\#series=OPTICFIBRESUBSPC.

World Economic Forum (2019b): 6.05 Digital skills among active population. Verfügbar unter https://reports.weforum.org/global-competitiveness-report-2019/competitivenessrankings/?doing_wp_cron=1590397950.1625969409942626953125\#series=EOSQ508.

Bundesministerium für Wirtschaft und Energie (2020): Digitale Kompetenz der Gesellschaft steigt deutlich an. Verfügbar unter https://www.bmwi.de/Redaktion/DE/Pressemitteilungen/2020/20200225-digitale-kompetenzder-gesellschaft-steigt-deutlich-an.html. 
Preprints der Zeitschrift BIBLIOTHEK - Forschung und Praxis, 2020, AR 3347 Schenkschuck und Flecke

Dies ist ein Preprint $(\boldsymbol{c c})$ EY-NG-ND. Die endgültige Publikationsfassung erscheint beim Verlag De Gruyter unter https://www.degruyter.com/view/j/bfup

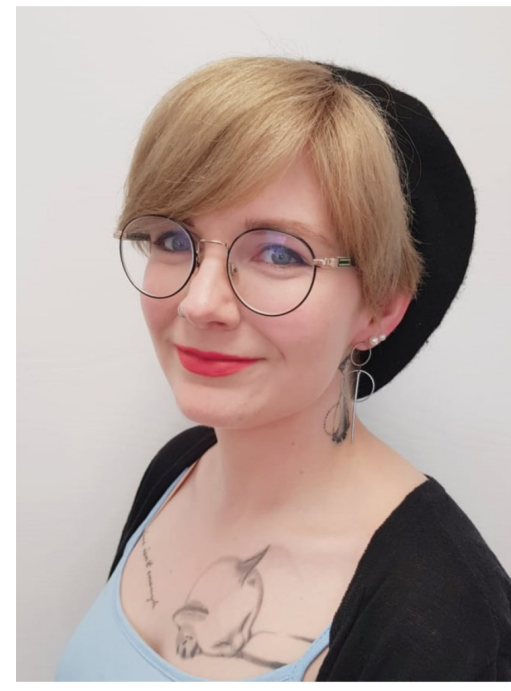

Annamarie Escher-Schenkschuck

Marburger Straße 10

D-57223 Kreuztal

a.escher@kreuztal.de

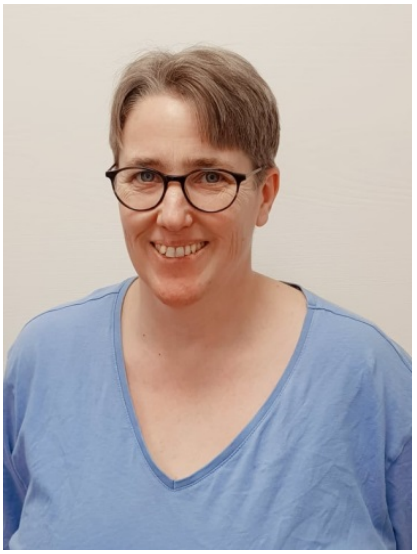

Sabine Flecke

Marburger Straße 10

D-57223 Kreuztal

s.flecke@kreuztal.de 